\title{
Les dessableurs de l'aménagement hydro-électrique de Dul Hasti (Inde)
}

\author{
par J. Binquet, D. Develay, Ph. Cazalis
}

Coyne et Bellier

\section{INTRODUCTION}

En octobre 1989, un consortium d'entreprises françaises s'est vu attribuer la conception, la construction et la mise en service du projet hydro-électrique de DUL HASTI en Inde pour le compte de la National Hydroelectric Power Corporation (NHPC), l'entité administrative indienne propriétaire du projet.

Coyne et Bellier, Bureau d'Ingénieurs-conseils est responsable de la mission d'ingénierie du projet (révision de l'avant-projet sommaire, préparation de l'avant-projet détaillé et élaboration des plans d'exécution) en collaboration étroite avec NHPC.

Une fois mis en service, cet aménagement sera, avec ses $390 \mathrm{MW}$, de puissance installée, l'un des plus grands aménagements hydro-électriques de l'Etat du Jammu et Cachemire. Le projet est situé sur la rivière Chenab non loin de la ville de Kishtwar dans la basse chaîne himalayenne. L'équipement de la chute de $236 \mathrm{~m}$, au droit de la boucle de la Chenab contournant le plateau de Kishtwar, consiste en la construction d'un barrage de dérivation en béton de $65 \mathrm{~m}$ de haut, d'une galerie d'amenée de $10,6 \mathrm{~km}$ avec prise d'eau et dessableurs en tête et d'une usine souterraine avec ses ouvrages connexes à l'aval (fig. l).

Une description complète de ce projet a fait l'objet de publications par Binquet et al. (1992) et Divatia et al. (1993).

$\mathrm{Au}$ droit du site du projet, la rivière Chenab a un transport solide particulièrement élevé durant la fonte des neiges et la période des crues. La composition minéralogique des particules en suspension révèle la présence de particules de quartz dont la fraction la plus grossière causerait du fait de sa dureté intrinsèque de sérieux dégâts aux directrices, aux roues et aux autres éléments hydromécaniques des turbines si on laissait passer ces particules abrasives dans le chemin d'eau.

Pour faire face à ces problèmes, on a retenu la solution consistant à réaliser un ensemble de dessablage au sein du complexe souterrain des ouvrages amont.

En raison de la topographie montagneuse du site, les dessableurs ont été conçus en ouvrages souterrains.

Ils comportent principalement deux chambres de dessablage du type Dufour, chacune traitant la moitié du débit total équipé. Il convient de signaler à ce propos que ce type

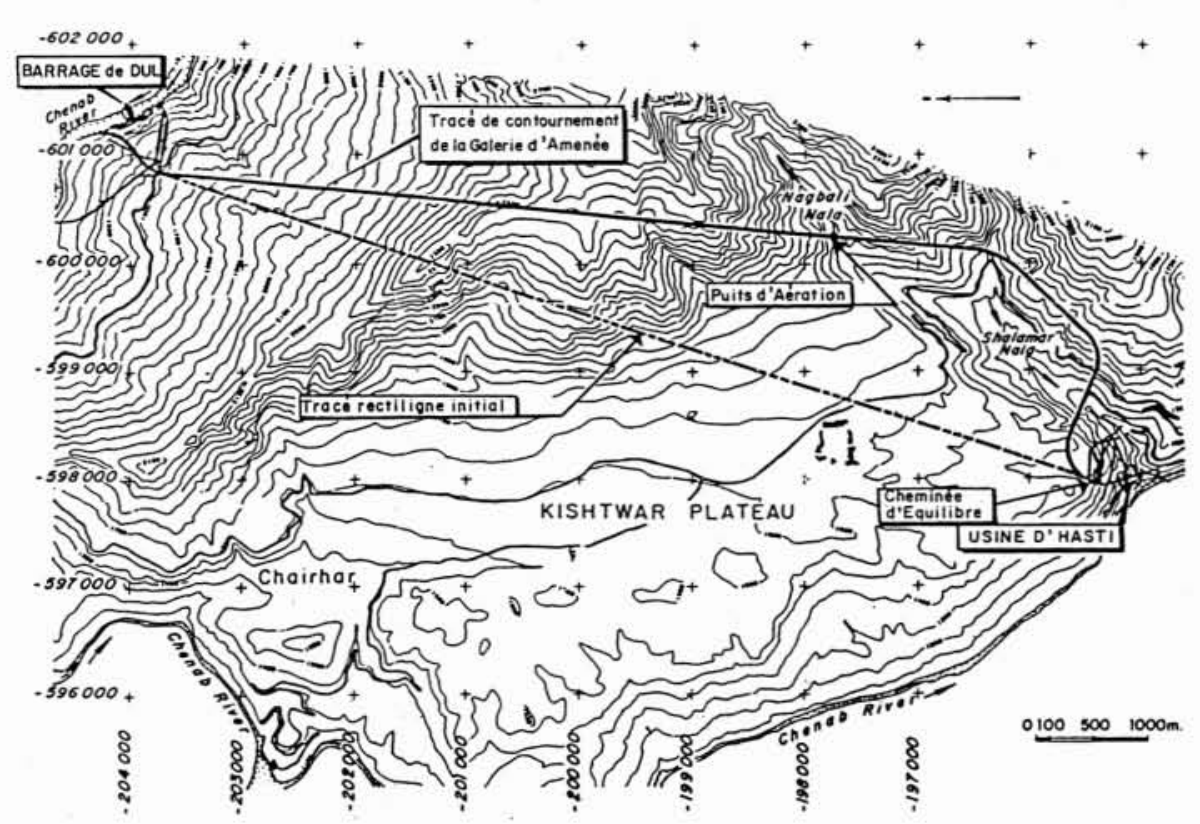

1. Vue en plan du projet Dul Hasti. 


\begin{tabular}{|c|c|}
\hline \multicolumn{2}{|r|}{ Notations } \\
\hline$Q_{i}$ & : débit capté à la prise d'eau $\left(\mathrm{m}^{3} / \mathrm{s}\right)$ \\
\hline$Q_{h}^{\prime}$ & débit de la galerie d'amenée $\left(\mathrm{m}^{3} / \mathrm{s}\right)$ \\
\hline $\mathscr{Q}_{f}$ & : débit du tunnel de chasse $\left(\mathrm{m}^{3} / \mathrm{s}\right)$ \\
\hline$\vec{C}$ & $\begin{array}{l}\text { : concentration sédiments en suspension } \\
\left(\mathrm{g} / \mathrm{m}^{3}\right)\end{array}$ \\
\hline$C_{i}$ & $\begin{array}{l}\text { : concentration sédiments en suspension à la } \\
\text { prise d'eau }\left(\mathrm{g} / \mathrm{m}^{3} \text { or } \mathrm{g} / \mathrm{l}\right)\end{array}$ \\
\hline$C_{f}$ & $\begin{array}{l}\text { : concentration sédiments en suspension dans } \\
\text { le tunnel de chasse }\left(\mathrm{g} / \mathrm{m}^{3} \text { or } \mathrm{g} / \mathrm{l}\right)\end{array}$ \\
\hline$C_{h}$ & $\begin{array}{l}\text { concentration sédiments en suspension dans } \\
\text { la galerie d'amenée }\left(\mathrm{g} / \mathrm{m}^{3} \text { or } \mathrm{g} / \mathrm{l}\right)\end{array}$ \\
\hline E & : rendement global du dessableur \\
\hline$E_{0.3}$ & $\begin{array}{l}\text { : rendement global du dessableur pour des par- } \\
\text { ticules de taille supérieure à } 0,3 \mathrm{~mm}\end{array}$ \\
\hline$D_{50}$ & $\begin{array}{l}\text { : diamètre moyen des particules de sédiment } \\
(\mathrm{mm})\end{array}$ \\
\hline$g_{s i}$ & $\begin{array}{l}\text { : taux de transport de matières solides déjau- } \\
\text { gées par unité de largeur }\end{array}$ \\
\hline$e_{b}$ & : facteur de rendement du charriage \\
\hline$h$ & : profondeur de l'écoulement $(\mathrm{m})$ \\
\hline$S$ & : pente de la ligne d'énergie \\
\hline $\tan _{\emptyset}^{\tan \alpha}$ & $\begin{array}{l}\text { coefficient de frottement de la matière solide } \\
\text { taille de la particule de sédiment }(\mathrm{mm})\end{array}$ \\
\hline$f c$ & : coefficient de frottement de Weisbach \\
\hline$n_{x}$ & : rapport d'échelle du modèle pour la grandeur \\
\hline$u$ & : vitesse du fluide $(\mathrm{m} / \mathrm{s})$ \\
\hline$U$. & : vitesse de frottement $(\mathrm{m} / \mathrm{s})$ \\
\hline$w_{s}$ & : vitesse de chute $(\mathrm{m} / \mathrm{s})$ \\
\hline$\sigma_{c}$ & $\begin{array}{l}\text { : facteur d'influence de concentrations plus } \\
\text { élevées }\end{array}$ \\
\hline K & : constante de Von Karman \\
\hline$\gamma_{w}$ & : densité du fluide \\
\hline$L^{\prime \prime}$ & : longueur de la chambre de dessablage $(\mathrm{m})$ \\
\hline
\end{tabular}

de dessableur conçu à l'origine par l'ingénieur suisse H. Dufour (1943, 1951 et 1954) a fait l'objet de réalisations sur ce modèle en Europe (Suisse, France, Italie) mais également aux USA (selon Dominy, 1966), en Inde et ailleurs dans le monde.

Le débit équipé de l'usine est de $213 \mathrm{~m}^{3} / \mathrm{s}$. Le débit nominal de chaque dessableur est de $122,5 \mathrm{~m}^{3} / \mathrm{s}$ dont $106,5 \mathrm{~m}^{3} / \mathrm{s}$ pour l'usine et $16 \mathrm{~m}^{3} / \mathrm{s}$ pour le dispositif de purge.

Le tunnel de chasse, d'une longueur de $1060 \mathrm{~m}$, restitue les sédiments piégés vers la rivière à environ $1100 \mathrm{~m}$ à l'aval du barrage.

Les dessableurs souterrains ainsi que leurs ouvrages connexes impliquent la réalisation de diverses galeries de service et de puits destinés tant au fonctionnement courant des unités de dessablage qu'à la maintenance et l'inspection de celles-ci. La figure 2 montre l'implantation de ces ouvrages amont.

L'ingénierie générale et détaillée de ces structures a nécessité tout d'abord un pré-dimensionnement des ouvrages sur la base de considérations purement théoriques, puis une confirmation sur modèle réduit hydraulique pour vérifier l'adéquation et la taille des bassins ainsi que les arrangements et détails du soutirage et de la purge des sédiments. Enfin, l'étude abordait également la question de l'optimisation de fonctionnement des dessableurs avec le minimum de perte d'eau particulièrement durant l'étiage de la Chenab.

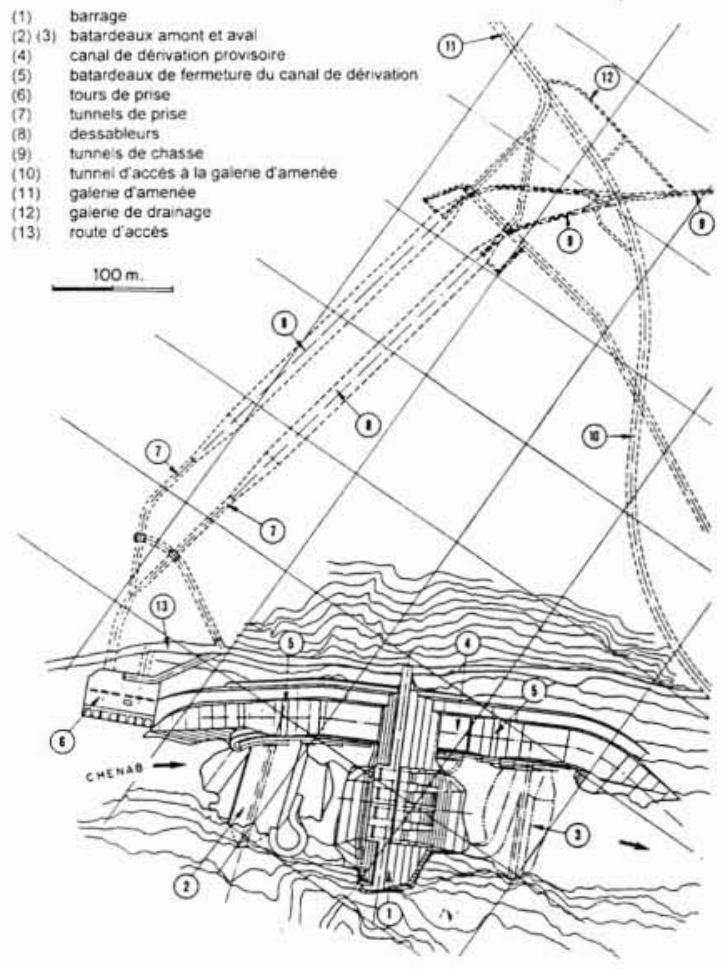

2. Vue en plan des ouvragres amont.

Cette publication relate en détail les études réalisées sur les thèmes ci-dessus et expose les améliorations obtenues à l'aide des applications sur modèle réduit hydraulique.

\section{HYDROLOGIE ET SÉDIMENTOLO- GIE}

La rivière Chenab prend sa source dans la région de Lahul/Spiti, dans la haute chaîne himalayenne, à une altitude d'environ $4900 \mathrm{~m}$. Sur le site du barrage, le lit de la rivière est à environ $1200 \mathrm{~m}$ d'altitude et présente une pente moyenne de 1 pour 80 .

Au droit du barrage, la surface de bassin versant totalise $10500 \mathrm{~km}^{2}$ dont $70 \%$ environ est sous couvert de neiges éternelles. Durant les mois de novembre à mars, c'est-àdire durant l'étiage, le débit de la rivière varie de 50 à $500 \mathrm{~m}^{3} / \mathrm{s}$. Durant les mois de juin à août, la période des hautes eaux, le débit varie de 600 à $3000 \mathrm{~m}^{3} / \mathrm{s}$ chaque année.

L'évacuateur de crue du barrage a été conçu pour une crue de projet de $8000 \mathrm{~m}^{3} / \mathrm{s}$.

Durant les basses eaux, l'eau de la rivière est très limpide et transporte fort peu de sédiments en suspension avec une concentration inférieure à $200 \mathrm{ppm}$. En revanche, durant la fonte des neiges et la période de crue, la rivière transporte une quantité de matériaux en suspension très importante et on estime le transport solide moyen annuel à environ 17 millions de mètres cubes.

La concentration normale en sédiments varie, durant la fonte des neiges et la période de crue entre 700 et $1500 \mathrm{ppm}$ et peut atteindre $50000 \mathrm{ppm}$ de façon exceptionnelle durant les très fortes crues. 
En tenant compte des deux régimes distincts évoqués ci-dessus, la conception particulière des dessableurs permet d'en garantir un fonctionnement optimal en prévoyant une chasse intermittente durant l'étiage et une chasse continue durant les hautes eaux de la mousson.

On trouvera dans le tableau $I$ les concentrations prises en compte dans l'étude et la conception des dessableurs, en présentation cumulée ou détaillée, en fonction de la taille des grains pour le cas normal ou le cas exceptionnel.

Tableau 1. - Concentration et tailles des particules des sédiments en suspension.

\begin{tabular}{|l|c|c|c|c|}
\hline \multirow{2}{*}{ Taille des particules } & \multicolumn{3}{|c|}{ Concentration des sédiments en suspension } \\
\cline { 2 - 5 } & \multicolumn{2}{|c|}{ Normal } & \multicolumn{2}{c|}{ Maximum } \\
\cline { 2 - 5 } & $g / \mathrm{L}$ & $\%$ & $g / \mathrm{L}$ & $\%$ \\
\hline $\begin{array}{l}\text { Matériaux grossiers } \\
(\phi>0,2 \mathrm{~mm})\end{array}$ & 0,1239 & 18,2 & 0,2403 & 17,3 \\
$\begin{array}{l}\text { Matériaux moyens } \\
(0,2>\phi>0,075 \mathrm{~mm}) \\
\begin{array}{l}\text { Matériaux fins } \\
(\phi<0,075 \mathrm{~mm})\end{array}\end{array}$ & 0,2363 & 34,6 & 0,4075 & 29,3 \\
\hline TOTAL & 0,3222 & 47,2 & 0,7428 & 53,4 \\
\hline
\end{tabular}

L'analyse minéralogique révèle que la plupart des particules en suspension sont de nature quartzitique. Par ailleurs, l'observation du tableau mentionné plus haut permet de constater que seulement la majeure partie des sédiments grossiers ainsi qu'une plus faible portion de la fraction moyenne de l'ensemble des matières en suspension captées par la prise d'eau seront piégées dans les dessableurs, tandis que le restant passera par les turbines et rejoindra la rivière au droit de l'ouvrage de restitution aval.

\section{ASPECTS GÉNÉRAUX DE LA CONCEP- TION DES ÉLÉMENTS DE PRISE D'EAU EN REGARD DU CONTRÔLE DU TRANSPORT SOLIDE}

Le réservoir créé par le barrage s'étire dans une gorge étroite sur environ $4,5 \mathrm{~km}$ dégageant une capacité utile très limitée (environ 8 millions de $\mathrm{m}^{3}$ ) calée entre le niveau de retenue normale $(1266,0)$ et le niveau minimum d'exploitation $(1238,9)$. En outre, la capacité de la tranche morte située en dessous du niveau minimum d'exploitation est très faible ( 3 millions de $\mathrm{m}^{3}$ ) et sera probablement comblée dès la première année d'exploitation.

La conception de la prise d'eau et de l'évacuateur de crue du barrage a été guidée par le souci majeur de limiter autant que possible la présence dans le chemin d'eau de matières solides transportées par la rivière. Les traits principaux de cette conception sont les suivants:
3.1 Des évacuateurs de crue du barrage à pertuis de fond surbaissés

Le seuil des blocs évacuateurs de crue est calé au plus bas au niveau 1225 .

L'évacuateur comprend quatre passes de section $9 \mathrm{~m} \times 9,5 \mathrm{~m}$ permettant une chasse du transport solide efficace afin de conserver la pérennité de la capacité utile de stockage (voir fig. 3).
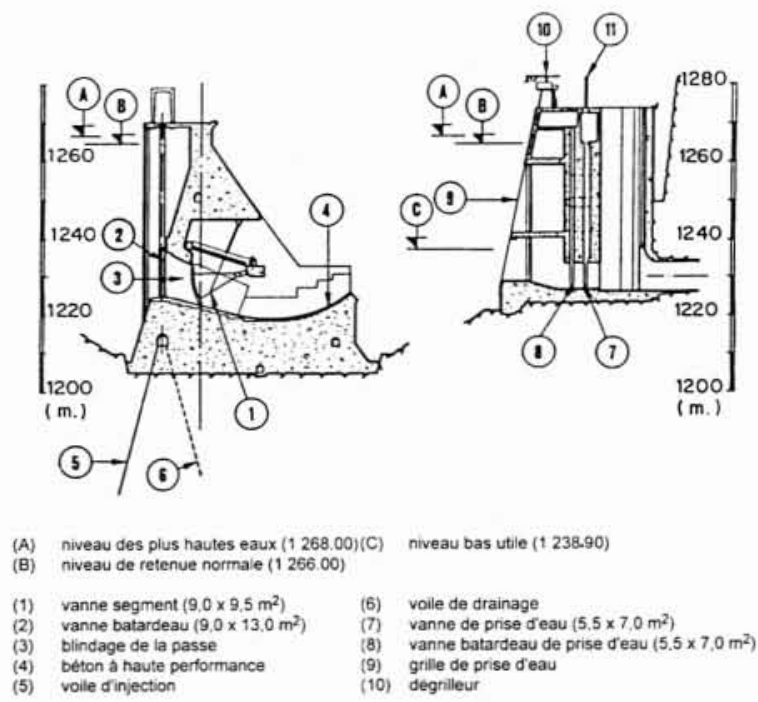

3. Coupe du barrage au droit de l'évacuateur de crue et coupe de la tour de prise d'eau.

\subsection{La prise d'eau}

La prise d'eau du conduit d'amenée est située sur la rive gauche, à proximité du barrage à environ $230 \mathrm{~m}$ à l'amont de l'axe de ce dernier. Une attention particulière a été portée sur la position de la prise d'eau dans une zone efficacement purgée par les chasses de l'évacuateur de crue.

La prise d'eau a une structure de tour semi-circulaire de $30 \mathrm{~m}$ de large, $40 \mathrm{~m}$ de long et $50 \mathrm{~m}$ de haut calée devant l'ouverture du tunnel de prise à section en fer à cheval de $7,4 \mathrm{~m}$ de diamètre. La tour comprend trois niveaux de prises vannées, chacune de dimension $5,5 \times 7,0 \mathrm{~m}$ aux niveaux $1226,50,1242$ et 1252 respectivement.

De cette façon seule l'eau de surface relativement peu chargée en matières en suspension est captée dans la prise de la prise d'eau durant la saison des crues, mais également durant les saisons intermédiaires et sèches, les prises étagées facilitant cette méthode d'exploitation jusqu'au niveau minimum utile à 1238,90 .

En face de la prise la plus basse au niveau 1226,50 , le radier s'élève au devant jusqu'au niveau 1229 , constituant ainsi un mur de protection s'opposant à l'entrée du charriage de fond dans le tunnel de prise (fig. 3 ). 


\subsection{Les dessableurs}

En complément des précautions évoquées ci-dessus pour éviter l'entrée des sédiments dans le tunnel de prise, on a prévu deux dessableurs souterrains de $15 \mathrm{~m}$ de large, $14,5 \mathrm{~m}$ de haut et $240 \mathrm{~m}$ de long placés immédiatement après la structure de prise pour tranquilliser et déposer les matières en suspension captées par la prise et les purger au moyen d'un dispositif de chasse comprenant un tunnel de chasse de $1060 \mathrm{~m}$ de longueur avec une section en $D$ de $4,5 \times 4,5 \mathrm{~m}^{2}$.

Les puits des vannes placés à l'amont et à l'aval des dessableurs permettent l'isolement, l'inspection et le nettoyage des chambres tout en conservant un fonctionnement partiel de l'aménagement. Le radier des chambres de dessablage est perforé d'orifices de soutirage de diamètre variant de 15 à $30 \mathrm{~cm}$ (sauf le premier de dimensions $0,5 \times 1,5 \mathrm{~m}^{2}$ ) et d'espacement variable longitudinalement (voir tableau 2) destinés à soutirer les sédiments déposés au moyen du collecteur de purge sous-jacent lui-même purgé par le tunnel de chasse. Les dimensions du collecteur de purge sous chaque chambre varient de $1,0 \times 1,5 \mathrm{~m}^{2}$ à $1,5 \times 3,0 \mathrm{~m}^{2}$. Chaque collecteur est prolongé par un tunnel de diamètre $2,6 \mathrm{~m}$, et les deux tunnels se rejoignent en un tunnel unique. Le débit de purge est contrôlé par une vanne à segment de dimensions $1,2 \times 1,8 \mathrm{~m}^{2}$.

Tableau 2. - Géométrie des orifices de soutirage des sédiments.

\begin{tabular}{|c|c|c|}
\hline Orifice & Dimensions & Position / Espacement \\
\hline $\begin{array}{l}\text { Premier orifice } \\
11 \text { orifices survants } \\
10 \text { onfices suvivants } \\
10 \text { orifices survants } \\
10 \text { orifices suivants } \\
10 \text { orifices suivants } \\
6 \text { orifices suivants } \\
16 \text { orifices survants } \\
\text { Dernier orifice }\end{array}$ & $\begin{array}{c}0.50 \mathrm{~m} \text { (targ) } \times 1.50 \mathrm{~m} \text { (iong) } \\
\$ 0.25 \mathrm{~m} \\
\$ 0.25 \mathrm{~m} \\
\$ 0.20 \mathrm{~m} \\
\$ 0.20 \mathrm{~m} \\
\$ 0.20 \mathrm{~m} \\
\$ 0.20 \mathrm{~m} \\
\$ 0.15 \mathrm{~m} \\
\$ 0.30 \mathrm{~m}\end{array}$ & $\begin{array}{l}\text { A l'extrémite amont } \\
1,50 \text { axe a axe } \\
2,00 \text { axe a axe } \\
2,50 \text { axe à axe } \\
3,00 \text { axe a axe } \\
3,50 \text { axe a axe } \\
4,00 \text { axe à axe } \\
5,00 \text { axe a axe } \\
\text { A l'extrémitè aval }\end{array}$ \\
\hline
\end{tabular}

\section{D CONCEPTION HYDRAULIQUE DES DESSABLEURS}

\subsection{Etudes préliminaires}

Le critère de dimensionnement du dessableur est de retenir puis de purger plus de $90 \%$ des sédiments dont le diamètre est supérieur à $0,3 \mathrm{~mm}(300 \mu \mathrm{m})$.

A l'occasion des études d'avant-projet sommaire effectuées par NHPC, les dimensions initiales des chambres étaient fixées à $300 \mathrm{~m}$ de long, 12,5 m de large et 14,5 m de haut. Le rendement de dessablage pour différentes tailles de particules a été étudié de façon théorique à l'aide du critère de Camp (1946). L'étude montre que pour un débit de $106 \mathrm{~m}^{3} / \mathrm{s}$ dans la galerie d'amenée et pour $8 \mathrm{~m}^{3} / \mathrm{s}$ dans le dispositif de chasse, le rendement de dessablage est de $98 \%$ et $87 \%$ pour des tailles de particules de $0,3 \mathrm{~mm}$ et $0,25 \mathrm{~mm}$ respectivement.

Par la suite, au cours de l'exécution du projet par le Consortium français, les dimensions des chambres ont été révisées en utilisant la méthode développée par Summer
(1977). En effet le critère de Camp ne tenait pas compte des effets du frottement contre les parois sur le transport des matières en suspension dans le bassin.

Au cours de cette révision furent étudiés les effets de deux valeurs de coefficient de frottement correspondant aux surfaces de béton coffré et béton projeté appliquées sur toute la longueur des chambres de dessablage y compris les zones de transition. Les résultats sont présentés dans le tableau 3.

\section{Tableau 3. - Estimation de la longueur des cham-} bres de dessablage.

\begin{tabular}{|c|c|c|}
\hline \multirow{2}{*}{$\begin{array}{c}\text { Paramètres considérès } \\
\qquad Q_{i}=122.5 \mathrm{~m}^{3} / \mathrm{s}\end{array}$} & \multicolumn{2}{|c|}{$\begin{array}{c}\text { Estimation de la longueur des chambres } \\
\text { de dessablage, } L(m)\end{array}$} \\
\hline & Alternative I & Alternative II \\
\hline $\begin{array}{l}\text { Surface de la section } \\
\text { Largeur de la chambre } \\
\text { Hauteur de la chambre }\end{array}$ & $\begin{array}{l}155 \mathrm{~m}^{2} \\
12,5 \mathrm{~m} \\
14.5 \mathrm{~m}\end{array}$ & $\begin{array}{l}170 \mathrm{~m}^{2} \\
15 \mathrm{~m} \\
14.5 \mathrm{~m}\end{array}$ \\
\hline $\begin{array}{l}\text { Vitesse de chute } \\
\text { Coefficient de frottement } \\
\text { fc }=0,009 \text { (beton coffré) } \\
\text { fc }=0,027 \text { (béton projeté) }\end{array}$ & $\begin{array}{l}L=318 \mathrm{~m} \\
L=338 \mathrm{~m}\end{array}$ & $\begin{array}{l}L=279 m \\
L=296 m\end{array}$ \\
\hline Vitesse de l'eau & $0,79 \mathrm{~m} / \mathrm{s}$ & $0,72 \mathrm{~m} / \mathrm{s}$ \\
\hline
\end{tabular}

(*) Pour des particules de $0,3 \mathrm{~mm}$.

A la lumière de ces résultats, on décida de poursuivre le projet par l'étude sur modèle réduit hydraulique d'un dessableur à parois de béton projeté de $300 \mathrm{~m}$ de long, $15 \mathrm{~m}$ de large et $14,5 \mathrm{~m}$ de haut (fig. 4).

Quelques modifications furent par ailleurs apportées à la conception initiale pour favoriser de meilleures opérations de soutirage et de purge des sédiments. Ces modifications furent les suivantes :

- raidissement des pentes des plans inclinés de la trémie inférieure,

- augmentation du débit de purge à $15 \mathrm{~m}^{3} / \mathrm{s}$ pour garantir une vitesse minimum de $3 \mathrm{~m} / \mathrm{s}$ dans le dispositif de purge, - séparation des écoulements du collecteur de purge et de la chambre de dessablage en ajoutant un radier perforé d'orifices de soutirage afin que les opérations de purge perturbent le moins possible le processus de sédimentation.

\subsection{Les essais sur modèle réduit hydraulique}

Les dessableurs de l'aménagement hydro-électrique de Dul Hasti ont été étudiés sur modèle réduit hydraulique :

- en 1987-88 à la Central Water \& Power Research Station à Pune en Inde (CWPRS) (1988). Ces essais ont permis à NHPC d'élaborer l'Avant-Projet sommaire,

- en 1990 au laboratoire d'hydraulique de Delft (LHD) (Hollande) sur la base de la conception révisée des bassins telle que décrite plus en détail par de Jong et al. (1992).

Les paragraphes qui suivent relatent essentiellement les études réalisées au LHD. Elles permirent la mise au point de l'Avant-Projet détaillé des dessableurs. On expose ci- 

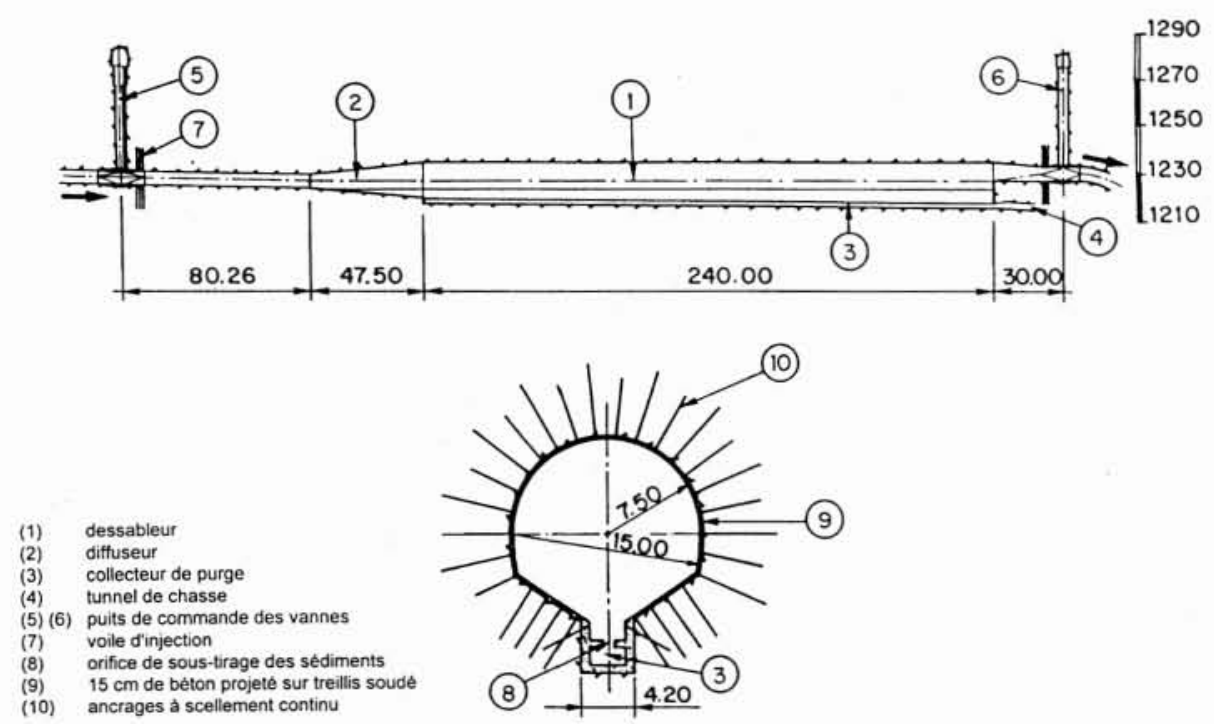

\section{Dessableur - profil longitudinal et coupe.}

après les quelques différences dans les approches techniques respectives des deux laboratoires CWPRS et LHD.

\subsubsection{Essais sur modèle réduit hydraulique au laboratoire d'hydraulique de Delft}

L'objectif de ces essais était d'une part d'optimiser la longueur des bassins tout en respectant les critères de dimensionnement (c'est-à-dire retenir dans les bassins et purger plus de $90 \%$ des sédiments dont le diamètre est supérieur à $0,3 \mathrm{~mm}$ ), d'autre part de réviser l'Avant-Projet sommaire du dispositif de purge proposé par CWPRS (taille et espacement des orifices dans le radier du bassin), enfin d'étudier les effets des purges en continu ou discontinues et en conséquence de proposer des modes d'exploitation adéquats particulièrement durant les périodes d'étiage.

\section{i) Analyse}

\section{- Echelles et conception du modèle}

Afin de limiter dans la mesure du possible les effets d'échelle on a décidé d'utiliser sur le modèle les matériaux de sédiment du prototype. Pour cette raison et du fait de considérations géométriques, le rapport d'échelle du rapport vitesse de l'eau sur vitesse de chute (U/Ws) devait être égal à l'unité $\left(n_{u / w_{s}}=1,0\right)$. Cette condition conduisait donc à adopter aussi un rapport d'échelle de vitesse égal à l'unité $\left(n_{u}=1,0\right)$.

En outre, les particules de sédiment étant en suspension, il était nécessaire d'avoir une distribution de concentration adéquate. Selon Rouse (1937) cette distribution de concentration peut se résumer dans l'équation suivante :

$$
\frac{C}{C_{b}}=\left(\frac{b}{h-b} \cdot \frac{\mathrm{h}-\mathrm{y}}{\mathrm{y}}\right)^{z}
$$

ou

$$
z=\frac{\sigma_{c} \cdot W_{s}}{K \cdot U_{*}}
$$

avec :

$C_{b} \quad=$ concentration au point de référence $y=b$

$h \quad=$ profondeur totale de l'écoulement

$\sigma \quad=$ facteur d'incidence de plus grande concentration sur le modèle comme sur le prototype (égal à 1)

$K=$ constante de Von Karman

$U_{*} \quad=$ vitesse de frottement

Avec un modèle géométriquement similaire (rapport d'échelle des longueurs $n_{t}=30$ ), on obtiendra un profil de concentration convenable à condition que :

$$
n_{W_{x} / U}=1
$$

avec

$$
n_{\sigma_{\sigma K}}=1
$$

Le développement de cette condition conduit à un rapport d'échelle de concentration $\left(n_{c}\right)$ égal à l'unité $\left(n_{c}=1\right)$.

Ce rapport d'échelle $\left(n_{c}=1\right)$ nécessite l'adoption du même coefficient de rugosité tant sur le modèle que sur le prototype.

Sur le prototype, la rugosité (béton projeté pour la section supérieure et béton coffré pour la section inférieure) donne une valeur selon Chezy de 70 (en tenant compte de l'effet des sédiments).

Sur le modèle, en utilisant des matériaux très lisses (Plexiglas et contreplaqué laqué) et en tenant compte de la rugosité des sédiments déposés sur le radier on a pu obtenir une valeur selon CHEZY de 65. Cette valeur légèrement inférieure correspondant donc à une rugosité un peu supérieure a conduit à développer sur le modèle des dunes de sédiment un peu plus hautes, ce qui est du côté de la sécurité. 
Considérant que les matériaux très fins ne peuvent que difficilement être piégés, on a retenu la solution de n'utiliser pour l'évaluation quantitative des performances du modèle, que les courbes granulométriques de la fraction des particules de taille supérieure à $0,075 \mathrm{~mm}$ avec un $D_{50}$ égal à $0,3 \mathrm{~mm}$.

Il fut toutefois vérifié que l'utilisation de matériaux plus grossiers n'avait aucune influence significative sur la forme des dépôts du modèle.

En ce qui concerne la conception du modèle lui-même, la figure 5 décrit le schéma retenu: l'eau et les matières solides en suspension du modèle circulaient dans un système en boucle fermée doté d'une pompe.

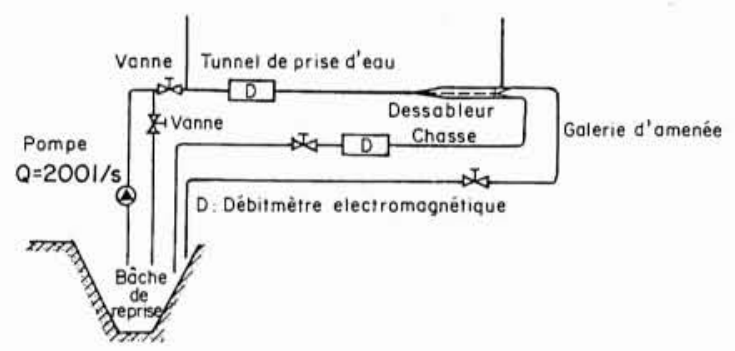

5. Schéma de principe du modèle hydraulique à boucle fermée.

\section{- Transition entre le tunnel de prise et le dessableur (diffu- seur)}

Il est apparu essentiel, pour optimiser le rendement du processus de sédimentation, d'attacher la plus grande importance à la distribution quasi uniforme des conditions d'écoulement sur toute la section en travers comme sur toute la longueur de la chambre de dessablage.

Les $20 \mathrm{~m}$ de longueur de transition avec une divergence de $1: 4,2$ et qui auraient conduit à une séparation de l'écoulement et à l'introduction d'un régime perturbé sur une longueur bien supérieure, à l'intérieur de la chambre de dessablage, ont fait l'objet d'une modification pour une transition sur une longueur de $47,5 \mathrm{~m}$ avec une divergence de $1: 10$. On a considéré qu'il s'agissait là du meilleur compromis pour l'optimisation des dimensions du dessableur.

\section{ii) Résultat des essais}

\section{- Purge en continu}

La concentration en matière en suspension $(C)$ dans la prise d'eau est définie comme suit :

$$
C_{i}=\frac{Q_{h} \cdot C_{h}+Q_{f} \cdot C_{f}}{Q_{i}}
$$

$i$ : prise d'eau

$h$ : galerie d'amenée

$f$ : tunnel de chasse

où $Q$ représente le débit.
Le rendement global du dessableur pour les particules de taille supérieure à $0,3 \mathrm{~mm}(E)$ est défini comme suit :

$$
E_{0,3}=1 \cdot \frac{Q_{h} \cdot C_{h}(\%>0,3)}{Q_{i} \cdot C_{i}(\%>0,3)}
$$

La figure 6 présente les principaux résultats obtenus avec des chambres de $300 \mathrm{~m}$ et $240 \mathrm{~m}$ de long.
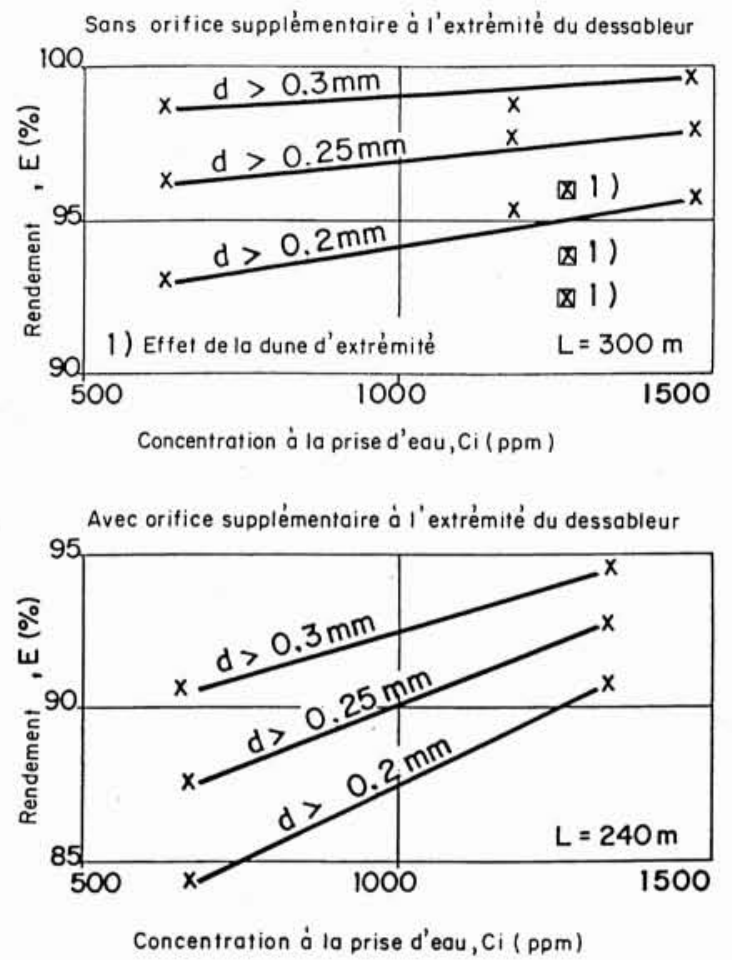

6. Rendement du dessableur en fonction de la concentration à la prise d'eau dans le cas d'une chasse continue.

Il apparaît que l'on obtient de meilleurs rendements lorsque la concentration augmente. Cela est dû au fait que la distribution des matières en suspension montre une concentration plus élevée dans la moitié inférieure d'une coupe verticale, ce qui fait que la concentration en sédiments dans le tunnel de chasse augmente plus rapidement que la concentration dans la galerie d'amenée. On a observé que même avec des concentrations extrêmement élevées (jusqu’à $5000 \mathrm{ppm}$ ) le rendement continuait d'augmenter.

Une chambre de dessablage plus courte présente de moins bons rendements qu'une chambre plus longue.

La chambre de dessablage de $240 \mathrm{~m}$ avec un diffuseur de $47,5 \mathrm{~m}$ de long a été retenue comme répondant de façon satisfaisante aux critères de dimensionnement imposés.

Il fut en outre observé qu'une dune se formait à proximité de l'extrémité aval de la chambre juste en dessous de point de séparation des eaux allant vers la galerie d'amenée. Les sédiments de cette dune pouvait aisément être entraînés dans cette galerie. Pour éviter cet inconvénient 
qui réduisait le rendement du dessableur, on a ajouté dans le radier un orifice supplémentaire d'un diamètre de $0,3 \mathrm{~m}$ placé à $6 \mathrm{~m}$ avant l'extrémité aval de la chambre. Mis à part cet orifice supplémentaire, le positionnement des autres orifices tel que prévu par CWPRS était réussi. On peut signaler tout particulièrement le débit de purge qui est suffisamment puissant pour offrir une capacité de transport des sédiments satisfaisante tout au long du collecteur inférieur grâce à l'orifice plus grand placé juste après le diffuseur.

Il est apparu enfin qu'avec un débit de purge réduit à $8 \mathrm{~m}^{3} / \mathrm{s}$ (soit $50 \%$ du débit de purge maximal) le dessableur est encore fonctionnel en dépit de quelques dépôts de sédiments dans le tunnel de chasse. Il fut donc recommandé de ne pas descendre le débit de purge en dessous de $13 \mathrm{~m}^{3} / \mathrm{s}$ car en deçà, lorsque la vitesse atteignait $3 \mathrm{~m} / \mathrm{s}$ les sédiments dans le tunnel de chasse étaient partiellement évacués par charriage.

\section{- Purge intermittente}

Lorsqu'à l'étiage de la rivière (octobre à avril) le débit capté devient chaque année bien inférieur au débit nominal de l'aménagement, il devient impératif pour des raisons d'optimisation d'usage de l'eau à des fins énergétiques d'en venir à un mode d'exploitation discontinu de la purge des dessableurs. On constate par ailleurs que pendant cette période la concentration en matière en suspension dans la rivière est bien plus faible.

On considéra dans ce cas deux processus distincts pour l'évaluation des performances de dessableur, à savoir :

- le processus de sédimentation,

- le processus de purge.

Durant la phase de sédimentation, c'est-à-dire lorsque les vannes de chasse sont fermées, les dunes commencent à se développer progressivement dans le temps et se propagent lentement à une vitesse d'environ $0,08 \mathrm{~m} / \mathrm{minute}$. Localement, avec la formation de dunes plus élevées, la vitesse augmente dans la chambre et progressivement la concentration en matière de suspension augmente dans le tube de la galerie d'amenée.

Pour une chambre de dessablage de $300 \mathrm{~m}$ de long, la formation et la propagation des dunes s'effectuait relativement vite et s'étendait sur environ $180 \mathrm{~m}$ après 20 heures de fonctionnement. Au-delà de 30 heures l'ensemble du collecteur de purge se trouvait comblé avec des collines de sable venant toucher la sous-face du radier. Enfin, au-delà de 50 heures, les dunes s'étendaient sur l'intégralité de la longueur de la chambre (avec cinq crêtes de dune régulièrement espacées le long de la deuxième moitié aval de la chambre) et la concentration en matière en suspension dans le tube de la galerie d'amenée augmentait de façon systématique jusqu'à deux fois la concentration initiale du début de l'essai.

Le nettoyage de la chambre s'effectue en utilisant le tube de chasse.

Dans le cas d'un collecteur de purge complètement rempli, seul l'orifice de sous-tirage aval commence à débiter à l'ouverture du tube de chasse. Après quelque temps, plusieurs orifices (situés du côté amont) commencent à débiter et le débit de purge augmente jusqu'à environ $13,5 \mathrm{~m}^{3} / \mathrm{s}$ évacuant tout le sable par le collecteur de purge dans le tube de chasse. Dans le cas où le collecteur de purge est rempli de telle façon que seules des colonnes de sable se forment sous les orifices, le sable du collecteur de purge est immédiatement chassé tout comme la plus grande partie des sédiments de la chambre. On a observé que quelques orifices restaient bloqués à cause de la formation de sortes de dômes au-dessus des orifices, dômes créés par le gradient élevé dans le sable durant la chasse. De tels dômes pouvaient être détruits par une fermeture suivie d'une réouverture rapide des vannes de chasse créant ainsi une onde de choc. Néanmoins ce n'était pas toujours efficace.

Manœuvrer la vanne de chasse de façon rapide (quelques minutes à l'échelle du prototype) et avec des fermetures de temps en temps peut permettre la purge du dessableur en deux heures environ.

Dans le cas où la vanne est manœuvrée lentement (1 à 2 heures à l'échelle du prototype), il était difficilement possible de purger complètement le dessableur à cause des effets de dôme même après quatre à cinq heures de manœuvre de chasse.

Le processus de remplissage dans le cas du modèle réduit est affecté par le choix des sédiments et par le rapport d'échelle. On s'attend notamment à ce que la hauteur de dune dans le prototype soit $30 \%$ de celle du modèle mais en contrepartie la formation de dunes surviendra aussi sur le prototype puisqu'il a été montré que la fraction fine des sédiments restait sans effet sur ce phénomène.

Afin de déterminer l'intervalle de temps entre les chasses, des observations ont été faites sur le modèle avec une concentration de $600 \mathrm{ppm}$ de particules de taille supérieure à la fraction tamisée ( $\phi>75$ microns). Dans la nature, la gradation inclut également des particules fines et lorsqu'on a recours à la chasse discontinue (durant l'étiage), les conditions de concentration en matière en suspension restent inférieures à $200 \mathrm{ppm}$.

Compte tenu du fonctionnement en période de pointe de l'aménagement hydro-électrique et de la sédimentation d'une fraction des matières en suspension dans le réservoir, on peut s'attendre à une réduction du taux de concentration des eaux captées à la prise.

Ainsi il est probable que la fréquence de chasse discontinue sera dans la pratique bien supérieure à 17 ou $20 \mathrm{~h}$ telle qu'estimée par l'observation du modèle. Sur le prototype on peut adopter provisoirement une fréquence de chasse intermittente bi-hebdomadaire qui sera modifiée par la suite en fonction de l'expérience réelle acquise. II convient de noter cependant que, parallèlement, l'intervalle de temps entre deux manœuvres de chasse ne peut pas être trop long pour éviter la tendance avec le temps, à la consolidation et au durcissement des sédiments déposés, ce qui peut conduire à de sérieux problèmes comme le blocage des orifices dû à des collines de sable consolidées dans le collecteur de purge

\subsubsection{Comparaison avec les essais sur modèle de CWPRS}

La différence essentielle entre les approches de CWPRS et LHD réside dans la représentation des matières en suspension.

A CWPRS on a employé, pour simuler les matières en suspension, de la poudre de noix écrasée calibrée et saturée ayant une densité de 1,4. 
La fraction des particules utilisées s'étalait sur une gamme allant de $0,06 \mathrm{~mm}$ à $6 \mathrm{~mm}$ avec un $D_{50}$ à $0,24 \mathrm{~mm}$.

Il fut estimé que le rendement global de dessablage pour la fraction de sédiment et pour la gamme de concentration testée dans le modèle variait entre 86,3 et $87 \%$, ce qui était en assez bonne concordance avec les $83,4 \%$ trouvés avec le critère de Camp. Après ajustement nécessaire sur la densité et les effets de modélisation, le rendement de dessablage était évalué à $96 \%$ sur la base d'une chambre de $300 \mathrm{~m}$ de long et pour différentes tailles de particules au-dessus de $0,3 \mathrm{~mm}$ à l'échelle du prototype.

\subsection{L'évacuation des sédiments}

A l'aval des dessableurs les sédiments déposés sont chassés vers la rivière par l'intermédiaire d'un tunnel de chasse unique de $1060 \mathrm{~m}$ de long fonctionnant à surface libre (pour les $32 \mathrm{~m}^{3} / \mathrm{s}$ des deux dessableurs).

$\mathrm{Si}$ l'on se penche sur le problème de l'évacuation des sédiments il est essentiel de vérifier l'adéquation de la capacité de transport avec la quantité estimée de sédiments déposés comme le souligne Avery (1989).

Le taux de transport de sédiment a été estimé en utilisant la formule de Bagnold applicable pour le transport solide total et citée par Graf (1971).

$$
g_{s i}=\gamma_{w} \cdot \text { h.s.u. }\left(\frac{e_{b}}{\tan \alpha}+\frac{0,01 \cdot u}{W_{s}}\right)
$$

où $g_{s i}=$ taux de transport de matière solide déjaugée par unité de largeur

$e_{b} \quad=$ facteur de rendement du charriage

$h \quad=$ profondeur de l'écoulement : $2,2 \mathrm{~m}$

$s \quad=$ pente du radier : 0,003

$u \quad=$ vitesse moyenne du fluide $: 3 \mathrm{~m} / \mathrm{s}$

$\tan \alpha=$ coefficient de frottement de la matière solide.

Pour des particules de diamètre moyen égal à $0,3 \mathrm{~mm}$, le taux de transport peut être estimé à $90 \mathrm{~kg} / \mathrm{s}$ pour un tunnel de chasse de $4,5 \mathrm{~m}$ de large, ce qui correspond à une concentration assez élevée d'environ $4,0 \mathrm{~g} / \mathrm{l}$ dans l'eau pénétrant dans le dessableur.

L'établissement du taux de transport de sédiment montre que la vitesse adoptée dans le tunnel de chasse est suffisamment élevée pour garantir un auto-curage du tunnel.

\section{V — NÉCESSITÉ D'ENTRETIEN ET CONSÉ- QUENCES SUR LA CONCEPTION DES OUVRAGES}

Pour des questions d'entretien, le Maître d'Ouvrage exige qu'il soit possible de vidanger l'un des deux dessableurs pendant que l'autre continue à fonctionner. Cette opération est rendue possible en manœuvrant à la fermeture la vanne amont ainsi que le batardeau aval du dessableur à inspecter.

Dans ce cas de figure, le phénomène de percolation d'un dessableur à l'autre mérite d'être contrôlé sans quoi les opérations de maintenance et d'entretien dans le dessableur vidangé risquent d'être fort difficiles. On a en effet :

— un revêtement du dessableur en béton projeté armé qui reste perméable.

- une distance d'entre-axe des dessableurs de $45 \mathrm{~m}$ seulement.
Ainsi il pourrait être nécessaire d'améliorer l'étanchéité du rocher périphérique aux dessableurs de façon à limiter le débit percolant durant les opérations de maintenance et selon la perméabilité naturelle du rocher à la périphérie de l'excavation, des injections de consolidation pourraient s'avérer indispensables.

Afin d'évaluer cet aspect d'une manière quantitative c'est-à-dire en évaluant le débit percolé dans le dessableur vidangé en fonction de la perméabilité du rocher et de la profondeur de l'anneau d'injection, une étude spécifique aux éléments finis a été effectuée pour simuler la percolation dans la masse rocheuse, en utilisant le logiciel GEFECOUL développé par Coyne et Bellier.

Le problème a été traité en bidimensionnel, la section considérée étant perpendiculaire à l'axe des dessableurs dont la section transversale est assimilée à deux cercles de diamètre $15 \mathrm{~m}$ (fig. 7).

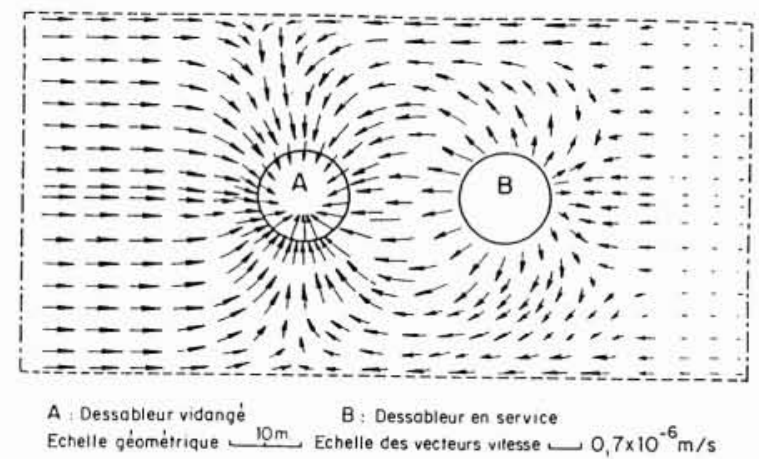

7. Flux de percolation vers le dessableur vidangé.

Le débit percolé dans le dessableur vidangé a été calculé pour différentes profondeurs d'injection ( $3 \mathrm{~m}$ et $8 \mathrm{~m}$ ) ainsi que sans injection du tout, en émettant l'hypothèse que la perméabilité du rocher injecté était de 5 unités Lugeon, soit $5 \times 10^{-7} \mathrm{~m} / \mathrm{s}$.

En ce qui concerne le rocher périphérique, trois valeurs de perméabilité ont été prises en compte, $10 \mathrm{UL}, 50 \mathrm{UL}$ et 100 UL (Unité Lugeon).

Les résultats de cette étude sont résumés dans le tableau 4

Tableau 4. - Estimation du débit de percolation vers le dessableur vidangé en fonction de la perméabilité du massif rocheux et de la profondeur des injections périmétrales des chambres.

\begin{tabular}{|c|c|c|c|}
\hline \multirow{2}{*}{$\begin{array}{l}\text { Hypotheses } \\
\text { de permeabilite } \\
\text { du massif rocheux }\end{array}$} & \multicolumn{3}{|c|}{ Profondeur d'injection } \\
\hline & Nulle & $3 m$ & $8 \mathrm{~m}$ \\
\hline $\begin{array}{l}10^{-6} \mathrm{~m} / \mathrm{s} \\
10 \mathrm{LU}\end{array}$ & $\begin{array}{c}4.8 \mathrm{~L} / \mathrm{min} / \mathrm{m} \\
19 \mathrm{~L} / \mathrm{s}\end{array}$ & $4 \mathrm{Umin} / \mathrm{m}$ & $3,4 \mathrm{~L} / \mathrm{min} / \mathrm{m}$ \\
\hline $\begin{array}{c}5 \times 10^{-5} \mathrm{~m} / \mathrm{s} \\
50 \mathrm{LU}\end{array}$ & $\begin{array}{l}24 \mathrm{~L} / \mathrm{min} / \mathrm{m} \\
96 \mathrm{~L} / \mathrm{s}\end{array}$ & $\begin{array}{c}10 \mathrm{~L} / \mathrm{min} / \mathrm{m} \\
40 \mathrm{~L} / \mathrm{s}\end{array}$ & $\begin{array}{c}7 \mathrm{U} / \mathrm{min} / \mathrm{m} \\
28 \mathrm{~L} / \mathrm{s}\end{array}$ \\
\hline $\begin{array}{l}10.5 \mathrm{~m} / \mathrm{s} \\
100 \mathrm{LU}\end{array}$ & $\begin{array}{l}48 \mathrm{~L} / \mathrm{min} / \mathrm{m} \\
192 \mathrm{~L} / \mathrm{s}\end{array}$ & $\begin{array}{l}12,6 \mathrm{Lmin} / \mathrm{m} \\
50 \mathrm{~L} / \mathrm{s}\end{array}$ & $8 \mathrm{U} / \mathrm{min} / \mathrm{m}$ \\
\hline
\end{tabular}


On indique deux valeurs dans chaque case: la valeur supérieure est le débit percolé par mètre linéaire longitudinal de dessableur tandis que la valeur inférieure est le cumul total de débit de percolation sur une longueur de dessableur valant $240 \mathrm{~m}$.

En supposant que le débit maximum acceptable sur l'ensemble du dessableur vidangé ne dépasse pas $75 \mathrm{l} / \mathrm{s}$ durant les opérations de maintenance, le niveau d'injection requis peut se lire sur le tableau, en fonction de la perméabilité du rocher. Cette perméabilité doit être vérifiée par des essais d'eau avant le traitement du rocher.

\section{VI $\square$ CONCLUSIONS $\square$}

Seul un programme d'essais sur modèle réduit hydraulique détaillé et rigoureusement réalisé a permis d'obtenir la confirmation de l'évaluation des performances des dessableurs pour différents modes d'exploitation en relation avec les processus de sédimentation et de purge.

Les études théoriques de ce problème complexe ne peuvent en effet que fournir les grandes lignes de dimensionnement des éléments principaux composant les dessableurs et le recours aux études sur modèle réduit hydraulique est une nécessité pour appréhender plus clairement les performances probables du prototype.

A l'aide d'une série d'essais sur modèle, décrits dans le présent document on a démontré que pour un débit nominal de $123 \mathrm{~m}^{3} / \mathrm{s}$ un dessableur de $240 \mathrm{~m}$ de long équipé à l'amont d'un diffuseur de $48 \mathrm{~m}$ et présentant une section transversale de $170 \mathrm{~m}^{2}$ permet de retenir plus de $90 \%$ de particules de diamètre supérieur à $0,3 \mathrm{~mm}$ à condition qu'un dispositif de purge continue fonctionne avec un débit qui ne soit pas inférieur à $13 \mathrm{~m}^{3} / \mathrm{s}$ et de préférence égal à $16 \mathrm{~m}^{3} / \mathrm{s}$.

On a également démontré que le rendement du dessableur augmente avec la concentration en sédiments et ce, même pour une valeur très élevée de $5000 \mathrm{ppm}$. En outre la présence de particules fines de diamètre inférieur à $0,25 \mathrm{~mm}$ dans la matière en suspension n'a pas d'effet négatif sur les résultats.

Dans le cas où l'on adopte une purge discontinue, le rendement du dessableur est progressivement réduit durant le processus de comblement du bassin. Ainsi, avec une concentration de $600 \mathrm{ppm}$, le rendement chute de $91,5 \%$ juste avant la fermeture du tunnel de chasse jusqu'à $75 \%$ après 17,5 heures (prototype) de fonctionnement sans purge. Ces résultats permirent de déterminer la fréquence des manœuvres de purge non seulement durant la mousson mais également durant l'étiage. Cette règle de gestion qui est initialement basée sur les essais sur modèle réduit sera ultérieurement modifiée grâce à l'expérience réelle acquise durant le fonctionnement de l'aménagement en affinant progressivement la règle au fur et à mesure de l'acquisition de données nouvelles.

\section{Références}

AWERY P. (1989) - «Sediment control at intakes. - A Design guide ». BHRA Process Engineering Division, The Fluid Engineering Centre, Bedford, England, 1989.

Binquet J., Develay D., Divatia E. and Venkatesha C.R. (1992). - « The Challenges of the Dul Hasti Project. Water Power \& Dam Construction, Oct. 1992.

CAMP T.R. (1946). - « Sedimentation and the design of settling tanks». Transaction ASCE, Vol. III, 1946, Paper No. 2285.

Central Water \& Power Research Station (1988). — « Hydraulic Model Studies for Dul Hasti Desilting Basin ", National Hydroelectric Power Corporation, Specific Note No. 2501, Feb. 1988 (unpublished).

COYNE et Bellier (1990). - « Final Design Report $»$. National Hydroelectric Power Corporation, Vol. 3, Dec. 1990.

Delft Hydraulics Laboratory, (1990). — « Report on the Tests carried out on a hydraulic scale model of desilting basins of Dul Hasti ». Special Report No. Q1220, Coyne et Bellier, Dec. 1990 (unpublished).

Divatia E., Venkatesha C.R., Binquet J. and Colombet G. (1993). - « Design of $10.6 \mathrm{~km}$ long Headrace Tunnel of Dul Hasti Hydroelectric Project ». Proc. Conference Options for Tunnelling 1993, H. Burger Editor (Developments in Geotechnical Engineering, 74), Elsevier, Amsterdam, May 1993.

DOMINY F.E. (1966). - «Design of Desilting Works for Irrigation Systems ». Proc. ASCE, Journal of the Irrigation and Drainage Division, Vol. 92, No. IR4, Dec. 1966, pp. $1-26$.

Dufour H. (1943). - «Prise d'eau avec dégraveur et dessableur pour un débit de $100 \mathrm{~m}^{3} / \mathrm{s} »$. Bulletin Technique de la Suisse Romande, 2 octobre 1943.

Dufour H. (1951). - «Le dessableur de l'usine de LaveyDescription ». Bulletin Technique de la Suisse Romande, No. 1-13, janvier 1951.

Dufour H. (1954). - «Le dessableur de l'usine de LaveyRésultats d'exploitation de 1950 à 1953 ». Bulletin Technique de la Suisse Romande, No. 10, mai 1954.

Graf, WAlter H. (1971). - « Hydraulics of Sediment Transport». Mc Graw-Hill Series in Water Resources and Environmental Engineering, Mc Graw-Hill Book Company, New York.

Jong de R.J., Perdijk H.W.R., Develay D., Gautier J. and BINQuet J. (1992). - « Hydraulic Model Studies of Desilting Basins of a Hydroelectric Project ", Proc. 5th International Symposium on River Sedimentation, Karlsruhe, Germany.

Rouse H. (1937), - «Modern Conception of the Mechanics of Fluid Turbulence ». Transactions ASCE, No. 102, 1937.

SUMER MUTLU B. (1977). - « Settlement of solid particles in open channel flow ». Journal of Hydraulic Engineering Division, Proc. ASCE, Vol. 103, Nov. 1977, pp. 13231337. 